\title{
Processor operated correlator with applications to laser Doppler signals
}

\author{
Bisgaard, C.; Johnsen, B.; Hassager, Ole
}

Published in:

Review of Scientific Instruments

Link to article, DOI:

$10.1063 / 1.1137808$

Publication date:

1984

Document Version

Publisher's PDF, also known as Version of record

Link back to DTU Orbit

Citation (APA):

Bisgaard, C., Johnsen, B., \& Hassager, O. (1984). Processor operated correlator with applications to laser Doppler signals. Review of Scientific Instruments, 55(5), 737-742. https://doi.org/10.1063/1.1137808

\section{General rights}

Copyright and moral rights for the publications made accessible in the public portal are retained by the authors and/or other copyright owners and it is a condition of accessing publications that users recognise and abide by the legal requirements associated with these rights.

- Users may download and print one copy of any publication from the public portal for the purpose of private study or research.

- You may not further distribute the material or use it for any profit-making activity or commercial gain

- You may freely distribute the URL identifying the publication in the public portal

If you believe that this document breaches copyright please contact us providing details, and we will remove access to the work immediately and investigate your claim. 


\title{
Processor operated correlator with applications to laser Doppler signals
}

\author{
C. Bisgaard, B. Johnsen, and O. Hassager
}

Instituttet for Kemiteknik, Danmarks Tekniske Hojskole, DK-2800 Lyngby, Denmark

(Received 23 September 1983; accepted for publication 15 December 1983)

\begin{abstract}
A 64-channel correlator is designed with application to the processing of laser Doppler anemometry signals in the range $200 \mathrm{~Hz}$ to $250 \mathrm{kHz}$. The correlator is processor operated to enable the consecutive sampling of 448 correlation functions at a rate up to $500 \mathrm{~Hz}$. Software is described to identify a Doppler frequency from each correlation and the system is especially designed for transient flow signals. Doppler frequencies are determined with an accuracy of about $0.1 \%$.
\end{abstract}

PACS numbers: 06.50.Dc, $42.60 . \mathrm{Kg}, 47.80 .+\mathrm{v}$

\section{INTRODUCTION}

In recent years several types of correlators have been reported in the literature. The correlators have mainly been designed for measuring the spectra of radio astronomy signals ${ }^{1,2}$ and investigation of macromolecules in fluids. ${ }^{3-6}$

In the present paper an on-line correlator, especially designed for laser Doppler application is presented. The correlator is of the clipping type, where the input signal is quantizied to $1 \mathrm{bit}$, and the autocorrelation function is calculated in real time.

The correlator is incorporated with a microprocessor to form a unit that we call a processor operated correlator (POC). The POC is capable of storing 448 independent autocorrelations in each run. Each autocorrelation consists of 648 -bit values. The collected data is transmitted to a bigger computer for further analysis. A set of software routines has been developed for this purpose, including a fast Fourier transform programmed in assembler code for the INTEL 8088 processor.

In laser Doppler applications each autocorrelation is ultimately connected to an "instantaneous" velocity component so that the possibility of storing $\mathbf{4 4 8}$ consecutive autocorrelations makes the instrument very suitable for velocity measurements in oscillatory or transient flows. The correlator is constructed of commonly available electronic components and the total price of the unit is very low compared to commercially available correlators or spectrum analyzers.

\section{PROCESSOR OPERATED CORRELATOR}

The correlator is designed to compute the function

$$
R(j)=\sum_{i=j}^{N} x_{i} x_{i+j-1} \quad, \begin{aligned}
& i=1,2, \ldots N . \\
& j=1,2, \ldots n .
\end{aligned}
$$

$x_{i}$ can be either zero or one, because of the 1-bit quantization of the signal. We shall refer to the function $R(j)$ as the autocorrelation function. In our correlator we use the fixed value $n=64$ while a number of different values of $N$ may be chosen as explained in the following. A diagram of the correlator is shown in Fig. 1. The basic elements of a hardwired correlator $^{7}$ consist of one $n$-bit shift register, $n$ multipliers, and $n$ stores. The digital signal is loaded into the first shift register multiplied to the values of the $n$-bit shift register including the first shift register, and the results are added to the $n$ stores.
The total system is shown in Fig. 2. Note that the POC unit here is connected to a SIRIUS computer, but that it could easily be connected to any other similar computer. The "signal in" is a 1-bit quantization of any given signal, the autocorrelation of which we wish to determine. It is possible to select the shift frequency of the correlator $f_{s}$ in the interval from 4.2 to $500 \mathrm{kHz}$ as shown in Table I. We also define the time from the start of a certain autocorrelation to the start of the next autocorrelation as the "sampling time" $t_{s}$. The user may select a sampling time from 10 preadjusted values in the range $2 \mathrm{~ms} \leqslant t_{s} \leqslant 1024 \mathrm{~ms}$, as shown also in Table $\mathrm{I}$.

We define the "number of clockpulses" $r$ by $N=r n$, where $n=64$ is the number of shift registers and $N$ is the total number of bits loaded into the correlator. The limited number of bits ( 8 ) for the storing of the different values of the autocorrelation function $R(j)$ sets a maximum value that $N$ may have without the risk of overflow in the stores.

In our correlator we have chosen to let $r$ (which is user controlled) be an integer, $1 \leqslant r \leqslant 7$, i.e., $N$ can have the user controlled values $64,128, \ldots 448$. The ZX81 microprocessor is used for collecting and storing of the autocorrelations. The data is moved from the correlator to the internal memory in the ZX81. The transfer of 648 -bit values from the correlator to the ZX81 takes $1.2 \mathrm{~ms}$ and is handled by an assembler subroutine.

In choosing the value of $r$, the shift frequency $f_{s}$ and the sampling time $t_{s}$, one must satisfy the following condition:

$$
t_{s}>r 64 / f_{s}+1.2 \times 10^{-3} \mathrm{~s} \text {. }
$$

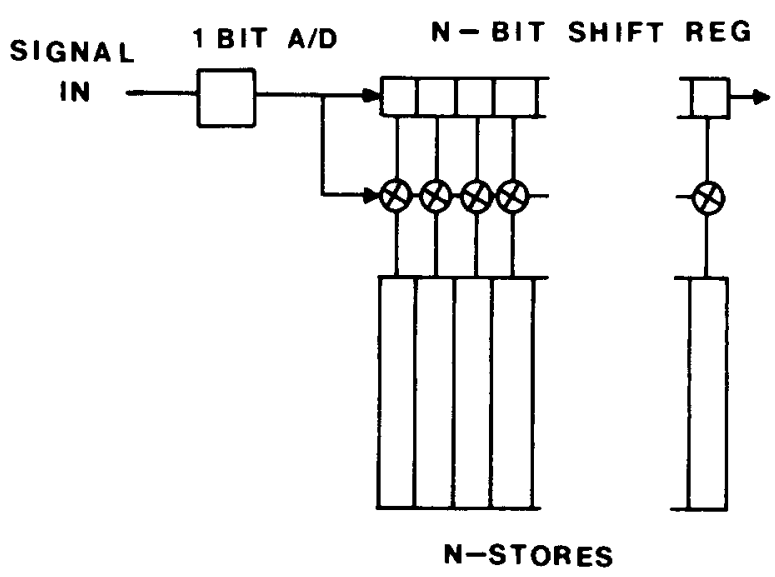

FIG. 1. Principles of the digital correlator 


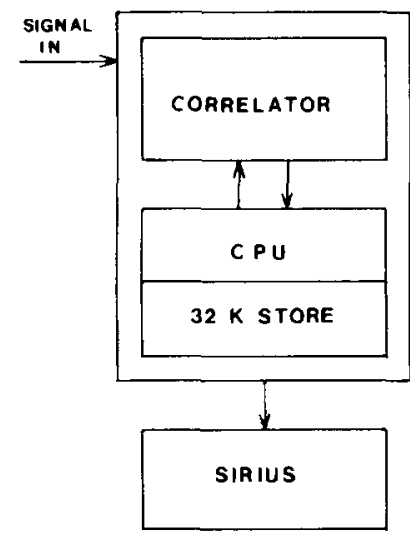

FIG. 2. Processor operated correlator and external computer.
The reason for this limitation is that it is not possible to sample faster than the correlator can compute and store one autocorrelation. In summary, the unit has three user-controlled parameters: $f_{s}, t_{s}$, and $r$. Table II summarizes the permissible values of $t_{s}$ for given values of $f_{s}$ and $r$.

Figure 3(a) shows the basic logic structure of the correlator. The clock signal (a) controls the shift frequency and time delay in the correlation and is created by an $8-\mathrm{MHz}$ master clock, which is divided by an integer value to create a suitable shift frequency. The data input on terminal (b) is moved into the first shift register and the count signal on terminal (c) multiplies the input data on terminal (b) with the instantaneous values in the shift register and the results are transferred to the 648 -bit counters. The data on terminal (b) is locked in the period beginning when the shift register is loaded and ending when the multiplication results have been transferred to the counters as shown in Fig. 3(b).

When $64 r$ bits have been loaded into the shift register and one autocorrelation has been computed and stored in 64 8-bit counters the data in the counters are transferred to latches, when the load signal on terminal (d) is activated. The data is transmitted to the data bus on the ZX81 over 64 8-bit three-state buffers, which are controlled by signals from the microprocessor.

When one autocorrelation is transmitted to the ZX81 the shift register and counters are reset to zero by a signal on terminal (e). The timing of the clock pulses, the load signal,

TABLE I. Available selections of shift frequency $f_{s}$ (and corresponding lag time $f_{s}^{-1}$ ) and sampling time $t_{s}$. The entry DIG is used as a label for the values of $f_{s}$ or $t_{s}$ selections.

\begin{tabular}{cccc}
\hline \hline DIG & $f_{s}(\mathrm{kHz})$ & $f_{s}^{-1}(\mathrm{~ms})$ & $t_{s}(\mathrm{~ms})$ \\
\hline 0 & & & 2 \\
1 & 500.0 & 2 & 4 \\
2 & 250.0 & 4 & 8 \\
3 & 125.0 & 8 & 16 \\
4 & 62.5 & 16 & 32 \\
5 & 31.3 & 32 & 64 \\
6 & 15.6 & 64 & 128 \\
7 & 7.8 & 128 & 256 \\
8 & 3.9 & 256 & 1024 \\
9 & & & \\
\hline \hline
\end{tabular}

TABLE II. Permissible minimum values of $t_{\mathrm{s}}$ for given values for $f_{\mathrm{s}}$ and $r$. The labels for $f_{s}$ and $t_{s}$ refer to the entry DIG in Table I

\begin{tabular}{|c|c|c|c|c|c|c|c|c|}
\hline \multirow[b]{2}{*}{$r$} & \multicolumn{8}{|c|}{$f_{s}(\mathrm{DIG})$} \\
\hline & 2 & 3 & 4 & 5 & 6 & 7 & 8 & 9 \\
\hline 1 & 0 & 0 & 0 & 1 & 1 & 2 & 3 & 4 \\
\hline 2 & 0 & 0 & 1 & 1 & 2 & 3 & 4 & 5 \\
\hline 3 & 0 & 0 & 1 & 2 & 2 & 3 & 4 & 5 \\
\hline 4 & 0 & 1 & 1 & 2 & 3 & 4 & 5 & 6 \\
\hline 5 & 0 & 1 & 1 & 2 & 3 & 4 & 5 & 6 \\
\hline 6 & 0 & 1 & 2 & 2 & 3 & 4 & 5 & 6 \\
\hline 7 & 0 & 1 & 2 & 3 & 3 & 4 & 5 & 6 \\
\hline
\end{tabular}

and the reset signal is shown in Fig. 3(c). A detailed description of the correlator and the interface to the ZX81 may be found elsewhere, ${ }^{8}$ including diagrams of the circuits, which generate the different load, reset, count, etc. signals to the correlator and a description of the interface from the ZX81 to the SIRIUS.

\section{SPECTRUM ANALYSIS}

The stored autocorrelations may be analyzed further for a number of different purposes. In this section we shall illustrate a method to obtain accurate estimates of the dominant frequency of each individual autocorrelation. These estimates are used in the analysis of laser Doppler signals for the purpose of measuring "instantaneous," "point values" of velocity.

The Fourier coefficients $Z_{k}$ are defined as follows:

$$
Z_{k}=\sum_{j=0}^{n-1} R(j) \exp \left(-i \frac{2 \pi j k}{n}\right) \text {. }
$$

Here $i=\sqrt{-1}$. In our situation there are $n=64$ real values of $R(j)$ (the imaginary parts are zero) and the complex $Z_{k}$ values contain 33 different real (cosine) coefficients and 31 different imaginary (sine) coefficients.

In order to estimate the desired frequency of the signal we first approximate the autocorrelation function by a cosine function of unknown frequency $(k+\epsilon) /(n \Delta t)$, where $k$ is an integer $1 \leqslant k<n / 2$ and $\epsilon$ is a fraction $0 \leqslant \epsilon \leqslant 1$. That is, we consider the following model for the autocorrelation:

$$
R(j)=\cos \left(\frac{2 \pi j(k+\epsilon)}{n}\right) ; j=1, \ldots . n .
$$

The resulting Fourier coefficients $Z_{k}$ and $Z_{k+1}$ are approximately

$$
Z_{k} \sim \frac{n \sin (\pi \epsilon)}{2 \pi \epsilon} e^{i \pi \epsilon}
$$

and

$$
Z_{k+1} \sim \frac{-n \sin (\pi \epsilon)}{2 \pi(1-\epsilon)} e^{i \pi \epsilon} .
$$

In connection with the derivation of these approximations we have found the following summation formula ${ }^{9}$ useful:

$$
\sum_{j=0}^{n-1} \cos (j y)=\cos \left(\frac{n-1}{2} y\right) \sin \left(\frac{n y}{2}\right) / \sin (y / 2) .
$$

From Eqs. (4) and (5) note that

$$
\operatorname{Re}\left(Z_{k}\right)>0, \quad \operatorname{Re}\left(Z_{k+1}\right)<0 \quad \text { for } 0<\epsilon<\frac{1}{2},
$$



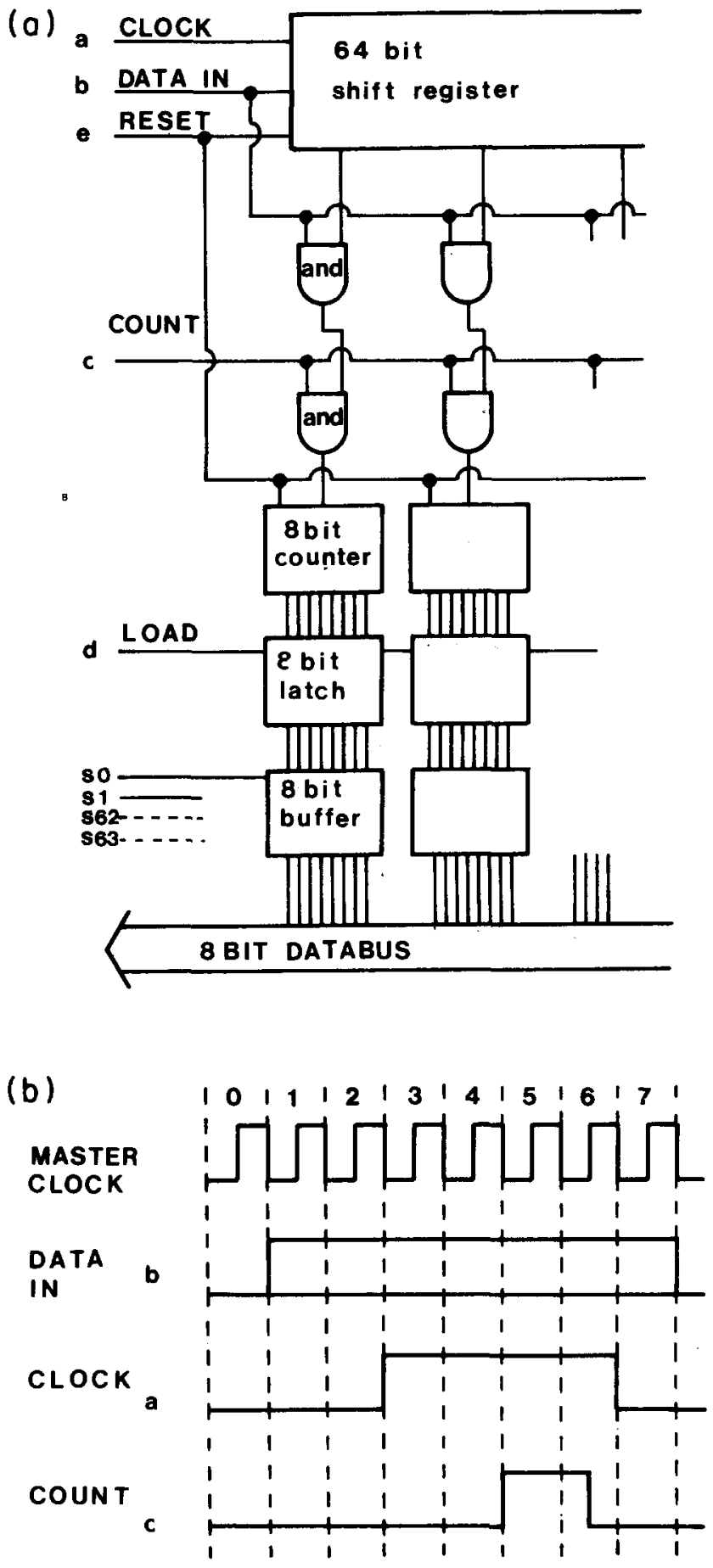

(c)
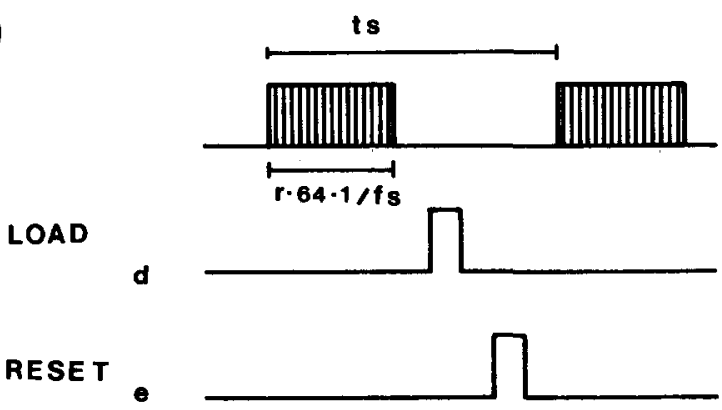

Fig. 3(a) Logic structure of the correlator, (b) control signals for computation, and (c) control signals for data transmission.

$$
\begin{array}{lll}
\operatorname{Re}\left(Z_{k}\right)<0, & \operatorname{Re}\left(Z_{k+1}\right)>0 & \text { for } \frac{1}{2}<\epsilon<1, \\
\operatorname{Im}\left(Z_{k}\right)>0, & \operatorname{Im}\left(Z_{k+1}\right)<0 & \text { for } 0<\epsilon<1 .
\end{array}
$$

In addition note that the imaginary parts dominate when $\epsilon \sim \frac{1}{2}$ while the real parts dominate when $\varepsilon \sim 0$ and $\varepsilon \sim 1$. Finally from Eqs. (4) and (5) note that the fraction $\epsilon$ may be determined from

$$
\epsilon=\frac{1}{1+\left(A_{k} / A_{k+1}\right)},
$$

where $A_{j}$ is the amplitude defined as the modules of $Z_{j}$, i.e., $\boldsymbol{A}_{j}=\left|\boldsymbol{Z}_{j}\right|$.

We consequently arrive at the following two-step procedure for determination of the desired frequency:

First the maximum amplitude for $0<j<m / 2$ is located. This amplitude may be associated either with frequency $k$ or $k+1$, and the final identification of $k$ is then based on the sign rule for the sine coefficients in Eq. (9). If the pattern in Eq. (9) is not followed, the correlation is discarded.

Second, if an interval $(k, k+1)$ has been located, $\epsilon$ is determined from Eq. (10).

This method for estimating the frequency is identical to that of Pike, ${ }^{7}$ except that we have found the sign rule for the sine coefficients to be more reliable than that for the cosine coefficients.

Figure 4 shows an example of a Fourier transform of an autocorrelation showing both cosine and sine coefficients to illustrate the rules in Eqs. (7)-(9).

The above method to estimate the dominant frequency of each individual autocorrelation has been implemented on the SIRIUS (with the INTEL 8088 processor). The entire data processing, including computation of the Fourier transform by a Sande-Tukey FFT algorithm, is performed by an assembler program. ${ }^{8}$ The run time is $73 \mathrm{~ms}$ for each transformation.

\section{APPLICATIONS}

In this section we consider three applications of the processor operated correlator. (1) To a signal of constant fre-

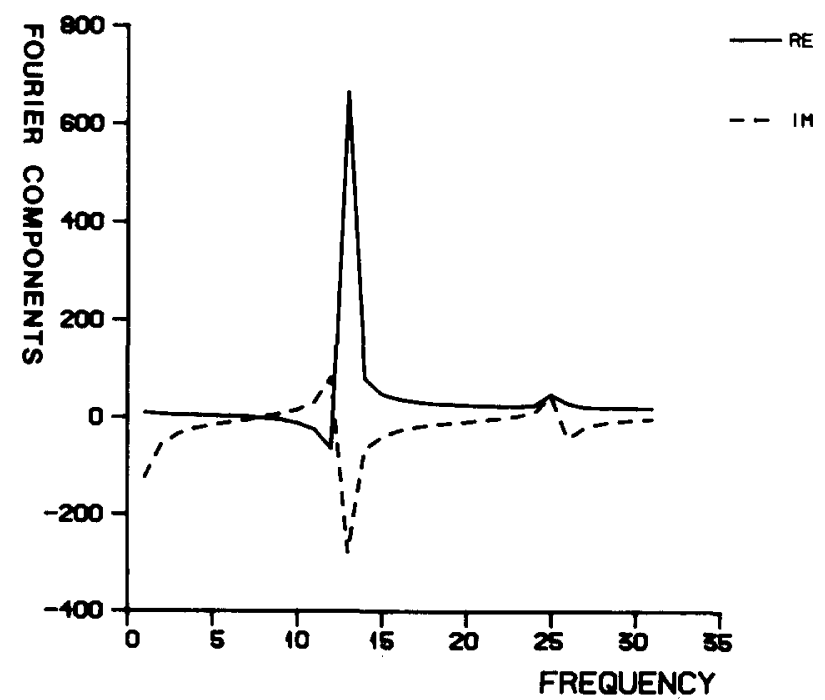

FIG. 4. Fourier transform of autocorrelation showing cosine (Re) and sine (Im) coefficients. Notice the second harmonic introduced by the 1-bit quantization. One unit on the abscissa is $f_{s} / 64$. 
quency and (2) to signals of variable frequency.

(A) The simplest application is the determination of the frequency of the signal from a frequency generator operating at a fixed frequency. Such a test is shown in Table III. The frequency generator was operated at seven different frequencies from 10 to $120 \mathrm{kHz}$, while the POC was operated with $f_{s}=250 \mathrm{kHz}$ and $t_{s}=8 \mathrm{~ms}$ in all tests.

The table shows mean values $F\left(=\Sigma_{i} F_{i} / 20\right)$ and standard deviations $S\left(S^{2}=\Sigma_{i}\left(F_{i}-F\right)^{2} / 20\right)$ of 20 single measurements $F_{i}(i=1, \ldots 20)$. The entry "Signal" is the result of a calibration of the signal with a pulse counter. Keep in mind, however, that the pulse counter has some uncertainty and that the frequency generator also has some drift. We see from Table III that there exists a frequency range in which the system determines a frequency with an accuracy better than $0.1 \%$. The upper limit of this range is given by the Nyquist or aliasing frequency $f_{s} / 2$. The lower limit depends on $r$ and is decreased with increasing $r$.

(B) The next application is the consecutive sampling of "instantaneous" values of the frequency of the signal from a frequency sweep generator shown in Figs. 5(a) and 5(b). The correlator samples with a frequency $f_{s}=250 \mathrm{kHz}$ and a sample time of 8 ms with $r=4$ to produce 448 "instantaneous" measured frequencies in a period of about $3.5 \mathrm{~s}$. The sweep generator was adjusted to produce a periodic sawtooth frequency modulated signal changing linearly with time in frequency from a low value to a high value with an instantaneous jump from the high frequency back to the low frequency. The output shown in Figs. $5(\mathrm{a})$ and $5(\mathrm{~b})$ is in agreement with what we expect based on the specifications of the sweep generator, but we have no independent way of measuring the frequency with an accuracy and dynamic response comparable to the present method. Keep in mind that each of the 448 estimated frequencies is based on independent autocorrelations and there is consequently no limitations to the allowed frequency change from one autocorrelation to the next as evidenced by the "instantaneous" drops in frequency. On the other hand there is a limitation on the extent to which the frequency is allowed to change within the duration of sampling of a single autocorrelation. We define the quantity $S_{1}$ as the rate of change of frequency at which the frequency of a given signal has changed by $f_{s} / 64$ during the sampling of one autocorrelation. With $64 r$ sampled bits at a rate of $f_{s}$ we find that $S_{1}=f_{s}^{2} /(4096 r)$. The signals in

TABLE I. Available selections of shift frequency $f_{s}$ (and corresponding lag time $f_{s}$ ') and sampling time $t_{s}$. The entry DIG is used as a label for the values of $f_{s}$ or $t$, selections.

\begin{tabular}{cccc}
\hline \hline $\mathrm{DIG}$ & $f_{s}(\mathrm{kHz})$ & $f_{s}{ }^{-1}(\mathrm{~ms})$ & $t_{s}(\mathrm{~ms})$ \\
\hline 0 & & & 2 \\
1 & & & 4 \\
2 & 500.0 & 2 & 8 \\
3 & 250.0 & 4 & 16 \\
4 & 125.0 & 8 & 32 \\
5 & 62.5 & 16 & 64 \\
6 & 31.3 & 32 & 128 \\
7 & 15.6 & 64 & 256 \\
8 & 7.8 & 128 & 512 \\
9 & 3.9 & 256 & 1024 \\
\hline
\end{tabular}
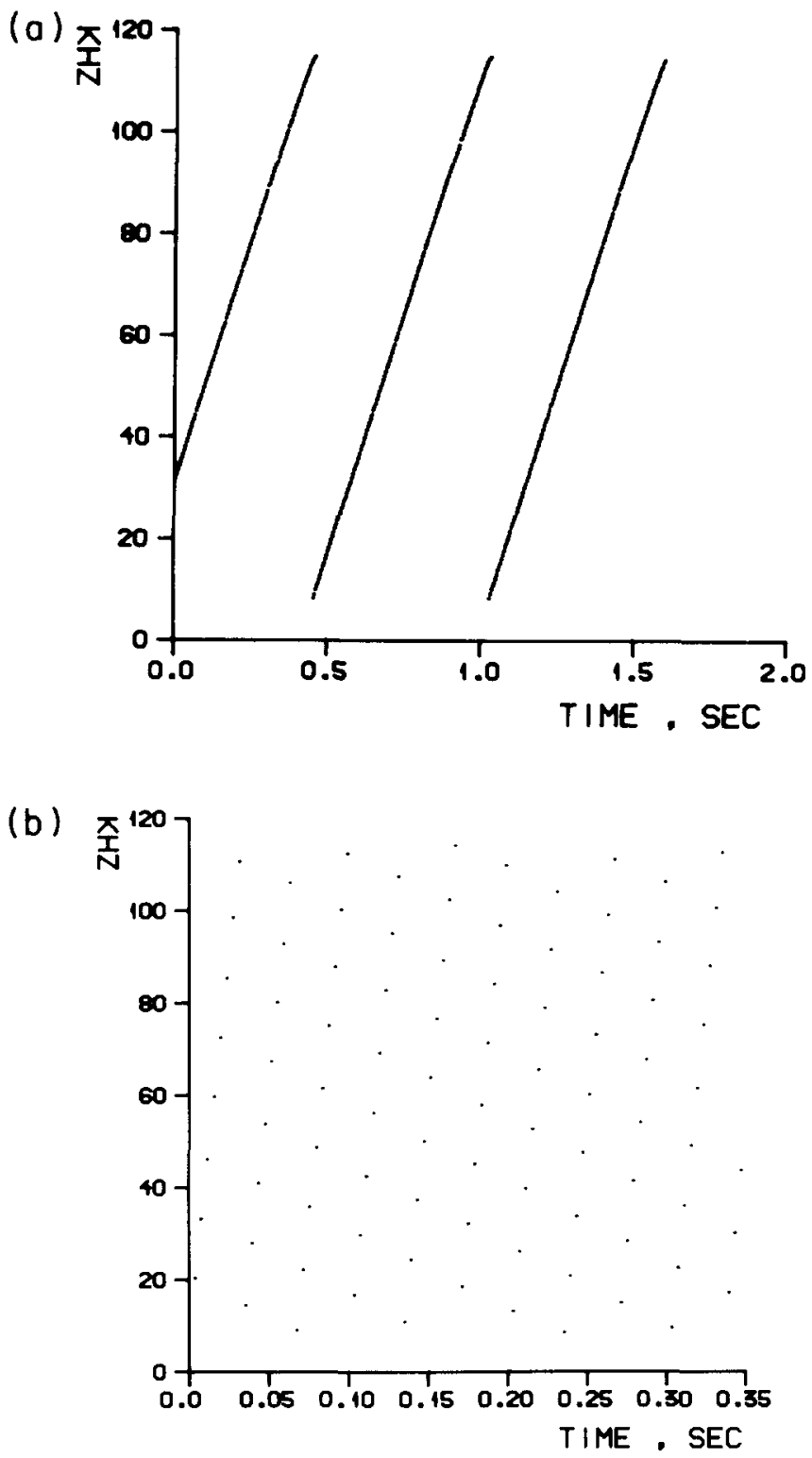

Fig. 5(a). Estimated frequencies of signal from sweep generator with sweep rate $0.18 \mathrm{kHz} / \mathrm{ms}$. Correlator: $f_{s}=250 \mathrm{kHz}, r=4, t_{s}=4 \mathrm{~ms}$ and (b) representation as in $5(\mathrm{a})$ but with sweep rate $3.5 \mathrm{kHz} / \mathrm{ms}$.

Figs. 5(a) and $5(\mathrm{~b})$ correspond to sweeps at rates of $(d f)$ $d t) \simeq 0.05 S_{1}$ and $(d f / d t) \simeq S_{1}$, respectively. Experimentshave shown that the correlator should not be used when $(d f / d t)$ exceeds $10 S_{1}$ since then the correlation begins to resemble that of white noise.

(C) The final application presented here is to the processing of signals obtained in laser Doppler anemometry (LDA). In its simplest form, LDA is a method to measure point values of one fluid velocity component from determination of the Doppler shift in frequency $f_{d}$, suffered by laser light when scattered by small particles present in the fluid. Consider two laser beams intersecting at a fixed point, the "measuring control volume." The measurable quantity is then the velocity component $v$ (at the measuring control volume) perpendicular to the bisector of the two beams and in the plane of the beams. The instantaneous value of $v$ is linearly related to the Doppler shift frequency $f_{d}$ as follows: 


$$
v=\left(f_{d}-f_{0}\right) \lambda /[2 \sin (\theta / 2)] .
$$

Here $\lambda$ is the wavelength of the laser light, $f_{0}$ is a "frequency shift," and $\theta$ is the angle of intersection of the laser beams. The signal of frequency $f_{d}$ is obtained from a photomultiplier focused at the measuring control volume.

The determination and recording of $f_{d}$ from the photomultiplier current is called Doppler signal processing. More details on LDA with particular emphasis on analog methods of signal processing may be found in Durst, Melling, and Whitelaw. ${ }^{10}$ For an alternative viewpoint with particular emphasis on digital processing of stationary signals see Cummins and Pike. ${ }^{7}$ In the following we shall illustrate the use of the processor operated correlator for processing of a transient Doppler signal.

In the geometry used here we consider a single sphere settling along the axis of a vertical cylinder filled with test liquid. The measuring control volume is located at a fixed point on the axis of the cylinder, and the axial component of liquid velocity is measured as a function of time. In the present example a polyacetal sphere of diameter $14.0 \mathrm{~mm}$ settles with a velocity of $21.3 \mathrm{~mm} / \mathrm{s}$ in a glycerine/water mixture contained in a tube of diameter $50.0 \mathrm{~mm}$. The correlator was operated to sample 448 correlations with a sample time $t_{\mathrm{s}}=16 \mathrm{~ms}$, a shift frequency $f_{s}=62.5 \mathrm{kHz}$, and $r=4$. The output in kilohertz is shown in Fig. 6. The flat part of the curves before and after the sphere passage correspond to zero liquid velocity.

During passage of the sphere through the measuring control volume there is no signal, and the corresponding correlations are rejected by the software. The present measurement is included only to illustrate the use of the correlator to the analysis of a "real" signal, the fluid mechanic interest in measurements of this particular kind being discussed elsewhere. ${ }^{11,12}$

\section{DISCUSSION}

We have described the development of a cheap processor operated correlator capable of on-line computations of autocorrelations with a shift frequency that can be adjusted in the range $4.2-500 \mathrm{kHz}$. A total of 448 individual autocorrelations are sampled and stored with a time interval that can be adjusted in the range from $2 \mathrm{~ms}$ to $1.024 \mathrm{~s}$ and the unit is ideally suited for the processing of transient signals. The unit was developed especially for the rather low-frequency signals that one typically obtains in LDA measurements in highly viscous or viscoelastic ${ }^{13}$ liquids, where analog processors cannot be used. For these applications a maximum shift frequency of $500 \mathrm{kHz}$ is usually sufficient but the design may be adapted to higher frequencies by the use of faster circuits. Alternatively one can precede the correlator with a fast storage and then use the correlator as a computational unit.

The correlator is especially designed to identify one dominating frequency in laser Doppler signals. Consequently the amplitude information lost in the 1-bit clipping of the signal is of no importance. The price paid for the increased computational speed gained by the 1-bit clipping is, however, a need for somewhat longer record lengths provided a constant statistical accuracy is desired. For more on 1-bit

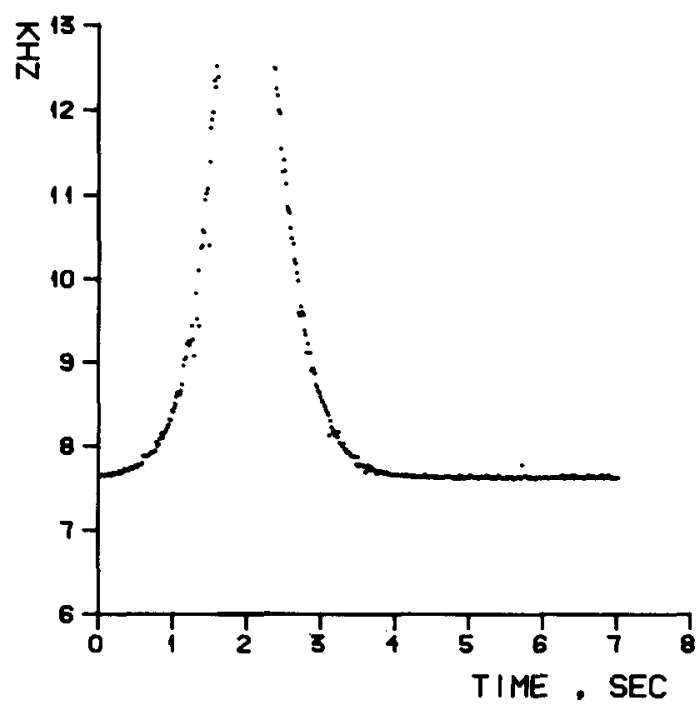

FIG. 6. Transient Doppler frequency from measuring control volume located at a fixed point on the axis of a vertical cylinder filled with glycerine. The liquid is forced to flow by a sphere settling along the axis of the cylinder, and the Doppler frequency is related to the instantaneous axial component of liquid velocity at the measuring control volume by Eq. (11) $(\lambda=632.8 \mathrm{~nm}$, $f_{0}=7.633 \mathrm{kHz}, \theta=9.52^{\circ}$ ).

clipping we refer to Otnes and Enochson. ${ }^{14}$

Frequencies may be determined in the complete range from $0.05 f_{s}$ to $0.5 f_{s}$. The accuracy is best in the high subrange from $0.1 f_{s}$ to $0.5 f_{s}$, namely about $0.1 \%$ for stationary signals. The accuracy in the low subrange from $0.05 f_{s}$ to 0.1 $f_{s}$ is dependent on $r$ and is increased with increasing values of $r$. The system should not be applied to frequencies above the Nyquist frequency $0.5 f_{s}$ since aliasing or folding errors are introduced in the computed frequency without warning. A simple check of this condition is to repeat a given measurement with a higher shift frequency.

The system treats each measurement individually and has remarkably good ability to follow transient signals as illustrated in Sec. III B. We have found a value of $r=4$ to be reasonable in all situations. However, in situations where extreme performance to follow sharp transient signals is needed one would use a lower $r$ value, while in situations where extreme noise rejection is needed one would use a larger $r$ value.

The distinct advantage of the present system compared to analog processing methods for LDA signals is the ability to store and retrieve individual autocorrelations and Fourier spectra for the user-controlled parameters: $f_{s}, t_{s}$, and $r$. This gives the possibility of a well-defined evaluation of the quality of each individual velocity measurement, a possibility not available with analog processing methods.

\section{ACKNOWLEDGMENTS}

The authors wish to acknowledge financial support from the Danish Council for Scientific and Industrial Research. We wish to thank $H$. Søeberg for useful advice on electronic circuits and M. L. Michelsen for a FORTRAN FFT code used in an early stage of the development. 
'J. G. Ables, B. F. C. Cooper, A. J. Hunt, G. G. Moorey, and J. W. Brooks, Rev. Sci. Instrum. 46, 284 (1975).

${ }^{2}$ S. Chopra and L. Mandel, Rev. Sci. Instrum. 43, 1489 (1972).

${ }^{3}$ R. Asch and N. C. Ford, Jr. Rev. Sci. Instrum. 44, 506 (1973).

${ }^{4}$ S. H. Chen, W. B. Veldkamp, and C. C. Lai, Rev. Sci. Instrum. 46, 1356 (1975).

${ }^{5}$ H. Sato and N. Suzuki, Jpn. J. Appl. Phys. 20, 401 (1981).

${ }^{6}$ N. S. Murthy and D. M. Choudhary, Rev. Sci. Instrum. 54, 476 (1983).

${ }^{7}$ E. R. Pike in Photon Correlation Spectroscopy and Velocimetry, edited by

H. Z. Cummins and E. R. Pike (Plenum, New York, 1977), pp. 246-343.

${ }^{8} \mathrm{C}$. Bisgaard, B. Johnsen, and O. Hassager, Internal Report, Instituttet for
Kemiteknik, DTH, Lyngby, Denmark, 1983.

${ }^{9}$ I. S. Gradshteyn and I. M. Ryzhik, Table of Integrals, Series, and Products (Academic, New York, 1965), item 1.341-3.

${ }^{10} \mathrm{~F}$. Durst, A. Melling and J. H. Whitelaw, Principles and Practice of LaserDoppler Anemometry (Academic, London, 1976).

"O. Hassager, Nature 279 (5712) 402 (1979).

${ }^{12}$ C. Bisgaard, J. Non-Newtonian Fluid Mech. 12, 283 (1983).

${ }^{13}$ R. B. Bird, R. C. Armstrong, and O. Hassager, Dynamics of Polymeric Liquids (Wiley, New York, 1977).

${ }^{14}$ R. K. Otnes and L. Enochson, Digital Time Series Analysis (Wiley, New York, 1972). 